\title{
AMP-activated protein kinase regulates autophagic protection against cisplatin- induced tissue injury in the kidney
}

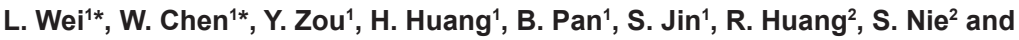
G. Kong ${ }^{1}$

${ }^{1}$ Department of Anesthesiology, Hunan Provincial People's Hospital, Changsha, Hunan, China

2Institute of Clinical Medical Research, Hunan Provincial People's Hospital,

Changsha, Hunan, China

${ }^{*}$ These authors contributed equally to this study.

Corresponding author: G. Kong

E-mail: gyk_hn@163.com

Genet. Mol. Res. 14 (4): 12006-12015 (2015)

Received January 28, 2015

Accepted May 29, 2015

Published October 5, 2015

DOI http://dx.doi.org/10.4238/2015.October.5.13

ABSTRACT. Although the nephrotoxicity of cisplatin has been well documented as a major side effect of chemotherapy, the exact mechanism by which prosurvival and apoptotic pathways interplay to determine renal pathology remains elusive. Recent studies suggested that autophagy might serve as an adaptive mechanism to promote cell survival during acute kidney injury $(\mathrm{AKI})$. We have used $\mathrm{AKI}$ as a disease model to investigate the mechanism regulating the cytoprotective role of autophagy in cisplatininduced tissue damage. Pharmacological inhibitors such as chloroquine were used to manipulate autophagy during AKI, and DNA damage was evaluated by using the cellular marker $\gamma \mathrm{H} 2 \mathrm{AX}$. Cisplatin induced extensive DNA damage during AKI. Autophagy activation served as a survival strategy to suppress cisplatin-induced DNA damage in the pathology of AKI both in vitro and in vivo. Interestingly, in the kidney, cisplatin treatment can activate AMP-activated protein kinase (AMPK), a signaling molecule that is also 
critical for p53-mediated inactivation of mammalian target of rapamycin (mTOR) pathways. As a result, inhibition or knockdown of AMPK can lead to repressed autophagy in cisplatin-induced AKI, resulting in more DNA damage. Activation of AMPK regulates autophagy during cisplatin-induced AKI. Given the fact that $p 53$ can regulate autophagy by inactivating mTOR via AMPK, our results suggest that the $\mathrm{p} 53$ pathway may also play a critical role in the pathogenesis of cisplatin-induced renal damage. This study may further our understanding of the physiological roles of autophagy in the pathogenesis of renal injuries, and thus may have pathological implications in the clinical setting.

Key words: Acute kidney injury; AMP-activated protein kinase; Cisplatin; Chloroquine; Autophagy; DNA damage

\section{INTRODUCTION}

Autophagy is a cellular process, activated in response to nutrient deprivation, by which the cell degrades dysfunctional or unnecessary cellular components for recycling of nutrients to promote survival and maintain cellular energy levels (Levine and Klionsky, 2004). The functions of autophagy are multidimensional: physiological levels of autophagy contribute to cellular energy homeostasis, while dysregulated autophagy has been implicated in pathological conditions such as cancer and acute injuries, uncontrolled autophagy may lead to cell death. Interestingly, recent studies have suggested that autophagy may serve as an adaptive mechanism to promote cell survival during acute kidney injury (AKI) (Pallet et al., 2008; Periyasamy-Thandavan et al., 2008; Yang et al., 2008; Bolisetty et al., 2010; Jiang et al., 2012).

AKI can be induced by multiple factors, including nephrotoxins, sepsis, and deficiency in the blood supply. The defining characteristic of AKI is renal stress, as a massive structural loss of renal tubules and cell death can be observed, resulting in damaged kidney functions. At the cellular level, apoptosis and necrosis account for cell death (Price et al., 2009; Basu and Krishnamurthy, 2010; Havasi and Borkan, 2011). Interestingly, autophagy may serve as a dichotomy point leading to two opposite cellular fates, i.e., promoting survival and inducing apoptosis. Therefore, dysregulation of autophagy is implicated in the pathogenesis of AKI (Sharfuddin and Molitoris, 2011). However, the regulatory mechanisms of autophagy during AKI remain unclear (Periyasamy-Thandavan et al., 2009). Given the cytoprotective role of autophagy in this process, AKI provides an excellent disease model for increasing our understanding of the pathological implications of autophagy in the clinic. In animal models of AKI, the clinically approved chemotherapeutic agent cisplatin is usually used to induce disease. Although the nephrotoxicity of cisplatin has been well documented as a major side effect of chemotherapy (Pabla and Dong, 2008; Miller et al., 2010), the exact mechanism by which the prosurvival and apoptotic pathways interplay to determine renal pathology is still elusive. Understanding the regulation of autophagy during cisplatin-induced AKI will help answer this question.

The purpose of the present study was to understand the regulation of autophagy by using cisplatin-induced AKI as a disease model. Both the in vivo and in vitro data from our study suggest that cisplatin induces extensive DNA damage during AKI. Interestingly, autophagy activation served as a survival strategy to suppress cisplatin-induced DNA damage in the pathology of AKI. Remarkably, such tissue injury caused by cisplatin was irreversible, since inhibition of autophagy by chloroquine could not alleviate the effect of cisplatin on renal damage. Furthermore, we identified 
a missing link that transduced the stress response to the regulation of autophagy during AKI: cisplatin treatment in the kidney activated the phosphorylation of AMP-activated protein kinase (AMPK), a signaling molecule that is also critical for p53-mediated inactivation of mammalian target of rapamycin (mTOR) pathways. Therefore, inhibition or knockdown of AMPK leads to repressed autophagy in cisplatin-induced $\mathrm{AKI}$, resulting in more DNA damage. The findings of this study confirmed the important role of autophagy in renal pathological processes. Most importantly, our discovery of the missing link between cisplatin-induced nephrotoxicity and autophagy highlights the significance of autophagic regulation in the pathogenesis of $\mathrm{AKI}$.

\section{MATERIAL AND METHODS}

\section{Materials}

Cisplatin and chloroquine were purchased from Sigma (USA). The AMPK inhibitor compound C [6-[4-(2-piperidin-1-yl-ethoxy)-phenyl)]-3-pyridin-4-yl-pyrrazolo[1,5-a]-pyrimidine] was purchased from Merck (USA). AMPK SMARTpool small interfering RNA (siRNA) was purchased from GE Dharmacon (USA). Primary renal proximal tubule cells were originally obtained from ATCC and maintained according to the culture conditions suggested by the provider. C56BL/6 mice were purchased from Jackson Lab. Antibodies against AMPK, p-AMPK, and p62 were purchased from Cell Signaling, andthe $\gamma \mathrm{H} 2 \mathrm{AX}$ antibody was purchased from Upstate Biotechnology.

\section{In vitro and in vivo models of cisplatin injury}

For in vitro experiments, cisplatin $(10 \mu \mathrm{g} / \mathrm{mL})$ and/or chloroquine $(25 \mu \mathrm{g} / \mathrm{mL})$ was added to cell cultures. For in vivo experiments, male C57BL/6 mice aged 8-10 weeks were injected with a single dose of $25 \mathrm{mg} / \mathrm{kg}$ body weight of cisplatin. Chloroquine was administered at a dose of 25 $\mathrm{mg} / \mathrm{kg}$ following injection of cisplatin. At different time points, the treated mice were sacrificed for collection of renal tissues. Renal morphological examination was performed by routine hematoxylin and eosin (H\&E) staining of the tissues. Animal studies were carried out under the supervision of a veterinarian, according to the guidelines for the use of laboratory animals of Hunan Provincial People's Hospital. All animals were provided by the Laboratory Animal Center of the Second Xiangya Hospital, and all protocols were approved by the Animal Care and Use Committee of Hunan Provincial People's Hospital.

\section{Western blot analysis}

Whole cells or tissues were homogenized and lysed, followed by protein quantification by the Bradford method, using a Bio-Rad protein assay kit. The proteins were resolved in NuPAGE ${ }^{\circledR}$ Novex 4 to $12 \%$ Bis-Tris precast gels, followed by electrotransfer to polyvinylidene fluoride membranes. The blots were incubated with primary and secondary antibodies before the final chemiluminescence reactions.

\section{Immunofluorescence}

Samples underwent serial dehydrations before being embedded in paraffin. Samples (4$\mu \mathrm{m}$ thick) were sectioned and subjected to immunofluorescence microscopy. 


\section{Knockdown of AMPK}

Cells were transfected with AMPK SMARTpool siRNA by using Lipofectamine 2000, following the manufacturer instructions (Invitrogen). After $72 \mathrm{~h}$, the cells were lysed and subjected to western blot analysis.

\section{Quantification of western blot results and statistical analysis}

Western blot results were quantified using Image (version 1.47). For in vivo studies, six mice were used for each treatment group, and the images (Figure 1) are representative of the experiments.

\section{RESULTS}

\section{Suppression of autophagy worsened cisplatin-induced AKI}

To evaluate the role of autophagy in cisplatin-induced AKI, we examined histological changes in the renal cortex in the absence or presence of chloroquine, a pharmacological inhibitor of autophagy. $\mathrm{H} \& \mathrm{E}$ staining of the renal cortex showed that cisplatin-induced AKI was exacerbated by treatment with chloroquine (Figure 1). At a dose of $25 \mathrm{mg} / \mathrm{kg}$, cisplatin induced moderate AKI $48 \mathrm{~h}$ after injection of the drug, as shown by a structural loss of the brush border, abnormal tubular distortion, and cell loss in proximal tubules (Figure 1, upper panels). In contrast, cotreatment with chloroquine significantly expedited the process. Histological changes of AKI became obvious as early as $24 \mathrm{~h}$ after coinjection of the drugs. Specifically, cotreatment with chloroquine caused elevated injury to the majority of the tubules, including tubular disruption and massive tubular lysis. The results suggest that activation of autophagy may serve as a protective mechanism to combat cisplatin-induced AKI.

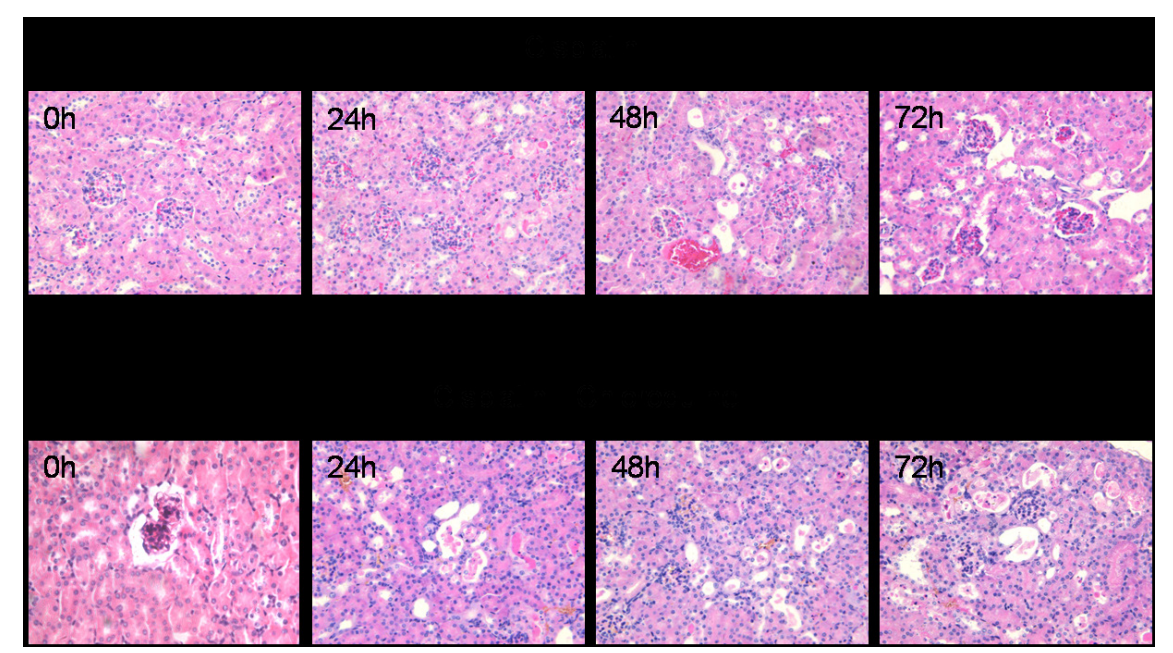

Figure 1. Activation of autophagy protected cells against cisplatin-induced AKI. Histological changes in the renal cortex in the absence or presence of chloroquine were determined by H\&E staining. At a dose of $25 \mathrm{mg} / \mathrm{kg}$, cisplatin induced moderate $\mathrm{AKI}$ at $48 \mathrm{~h}$ after injection of the drug. Cotreatment with chloroquine significantly expedited the process. Histological changes of AKI became obvious as early as $24 \mathrm{~h}$ after coinjection of the drugs. 


\section{DNA damage was concomitant with autophagy inhibition during cisplatin-induced AKI}

To further examine cisplatin-induced $\mathrm{AKI}$ at the molecular level, we measured the levels of the DNA damage marker $\gamma \mathrm{H} 2 \mathrm{AX}$ during cisplatin-induced AKI in the absence and presence of chloroquine. Indeed, cisplatin treatment led to increased levels of $\gamma \mathrm{H} 2 \mathrm{AX}$ (Figure 2A). We then examined the levels of p62, an autophagy substrate protein. p62 accumulated in the kidneys treated with chloroquine (Figure 2B). p62 is aubiquitin-binding protein and interacts directly with LC3 to facilitate autophagic degradation of ubiquitinated proteins (Bjorkoy et al., 2005). Therefore, the increased levels of p62 were indicative of the decreased autophagy activity caused by chloroquine treatment. Interestingly, concomitant with the declining autophagy activity, $\gamma \mathrm{H} 2 \mathrm{AX}$ levels stayed unchanged (Figure 2B). Thus, it became evident that, at the protein level, DNA damage was one of the major consequences following cisplatin treatment of mice; inhibition of autophagy by chloroquine did not mitigate the effect of cisplatin on $\gamma \mathrm{H} 2 \mathrm{AX}$. Taken together, the data show that cisplatin-induced AKI resulted in multidimensional damages: at the histological level, it caused a structural loss in renal tubules; and at the molecular level, DNA damage became evident. Chloroquine treatment abolished the protective function of autophagy during AKI, makingrenal damages irreversible.

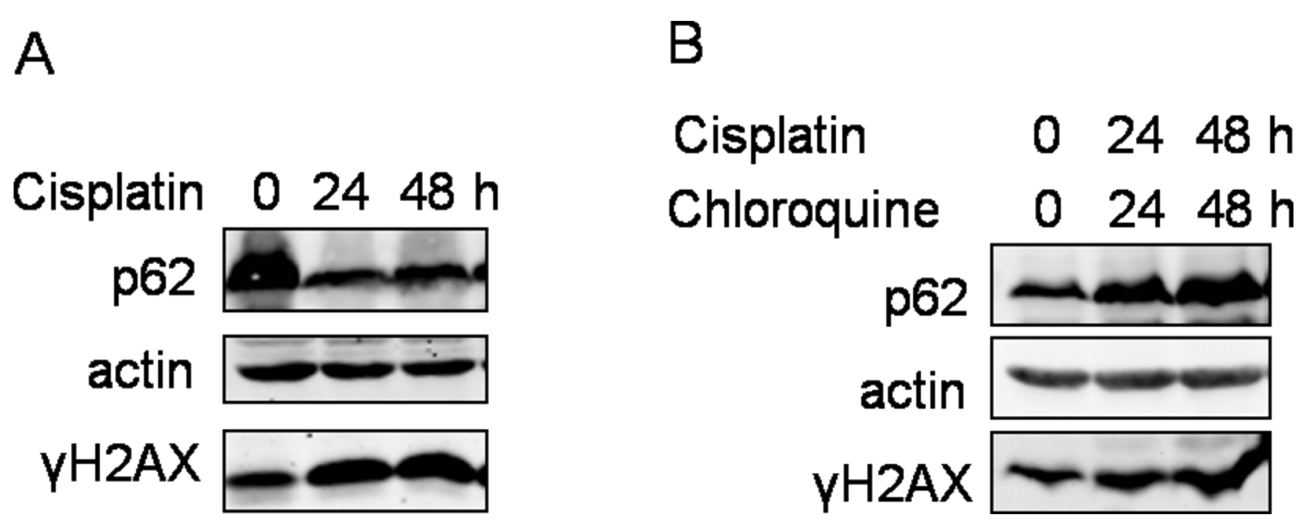

Figure 2. DNA damage could not be mitigated by autophagy inhibition during cisplatin-induced AKI. Cisplatin-treated mice were administered chloroquine (A) or did not receive chloroquine (B). At 48 and $72 \mathrm{~h}$ post-treatment, the kidneys were collected and subjected to western blotting to determine the levels ofthe autophagy marker p62 and the DNA damage marker $\gamma \mathrm{H} 2 \mathrm{AX}$.

\section{Chloroquine led to decreased autophagy activity in vitro}

To test whether chloroquine has an effect on autophagy in vitro similar to that observed in vivo, we evaluated the autophagy activity in primary kidney cells upon treatment with cisplatin in the absence and presence of chloroquine. In primary kidney cells, cisplatin treatment induced autophagy activity in a dose-dependent manner, as indicated by diminished levels of p62 (Figure $3 \mathrm{~A}$ ). The phenotype was confirmed by western blot analysis and immunofluorescence microscopy, by which increased LC3 II levels were clear (Figure 3C and D). LC3 can be processed and conjugated with phosphatidylethanolamine to form LC3 II, which is deposited on autophagic vesicles (Kabeya et al., 2000). Increased levels of LC3 II suggested an enhanced autophagy activity hours after cisplatin treatment (Figure $3 \mathrm{C}$ and D). Furthermore, the activity of autophagy 
could be reversed effectively by treatment with chloroquine, as indicated by enhanced levels of p62 and diminished levels of LC3 II in samples cotreated with chloroquine (Figure 3B and C). The results suggested that the primary kidney cells displayed similar effects of cisplatin-induced autophagy and that chloroquine could inhibit its activity, as we observed in the in vivo studies. Using this in vitro system, we proceeded to investigate the mechanism of regulation of autophagy activity during cisplatin-induced DNA damage, and we tried to understand the significance of this regulation at the cellular level.

A

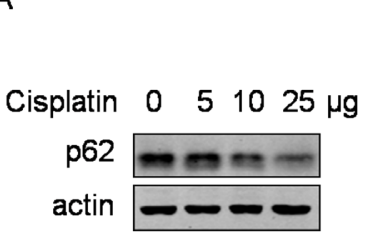

B

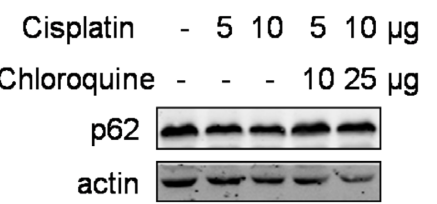

C

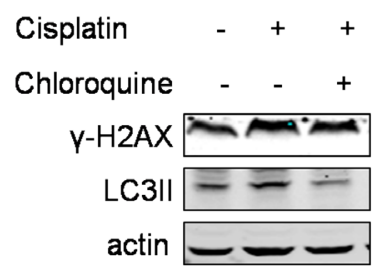

D
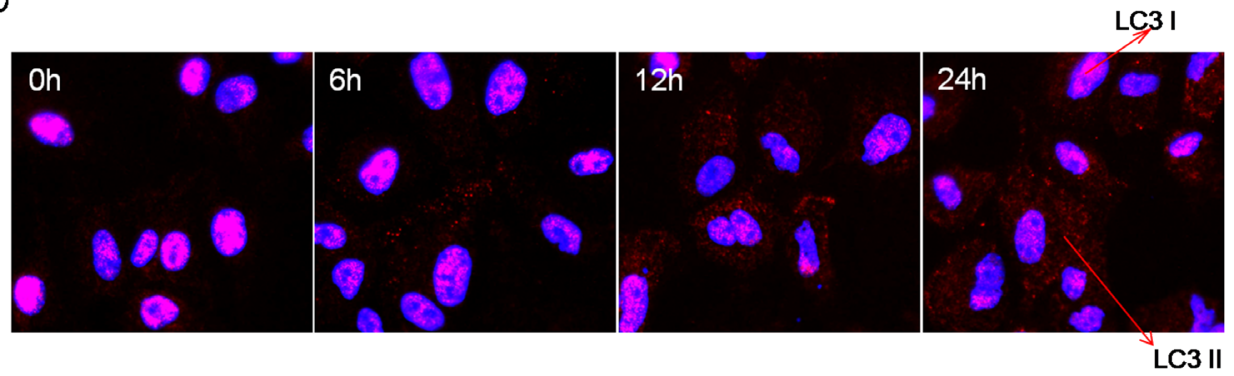

Figure 3. Chloroquine induced decreased autophagy activity in vitro. Primary kidney cells were treated with cisplatin in the absence $(\mathbf{A})$ or presence $(\mathbf{B})$ of chloroquine at the indicated concentrations. C. Effects of cisplatin and chloroquine on LC3 II levels were also evaluated in primary kidney cells. D. In vitro phenotype upon cisplatin treatment was examined by immunofluorescence microscopy for LC3 II and LC3 I staining.

\section{Cisplatin-activated AMPK during AKI}

To understand further the regulation of cisplatin-induced autophagy during $\mathrm{AKI}$, we examined signaling molecules that may mediate autophagy. In the p53-mediated autophagy pathway, phosphorylated AMPK is recruited for p53-mediated inactivation of mTOR (Feng et al., 2005). Interestingly, we discovered that the level of phosphorylated AMPK was significantly increased following cisplatin treatment (Figure 4A and B, lane 2). DNA damage was also obvious, as indicated by an increased level of $\gamma \mathrm{H} 2 \mathrm{AX}$ (Figure 4B, lane 2), consistent with previous observations. More importantly, inhibition of phosphorylated AMPK by compound $C$ significantly reduced autophagy activity, as indicated by elevated levels of p62, leading to exacerbated DNA damage $(\gamma \mathrm{H} 2 \mathrm{AX})$ (Figure 4B, lane 4). It is worth mentioning that treatment with compound $C$ alone did not cause severe DNA damage (Figure 4B, lane 3). To further confirm the role of AMPK, we knocked down the levels of AMPK by using an AMPK siRNA (SMARTpool), and we observed enhanced DNA damage in cells treated with cisplatin and siRNA (Figure 4C, lane 4). Strikingly, p62 levels were also 
elevated when AMPK was knocked down, suggesting a compromised autophagy activity (Figure $4 C)$. Taken together, the results suggest that AMPK plays a critical role in regulating cisplatininduced DNA damage. Impaired autophagy activity through inhibition of AMPK phosphorylation during AKI may lead to exacerbated DNA damage, and autophagic protection against cisplatininduced DNA damage is regulated by crosstalk between the p53 and mTOR pathways.

A

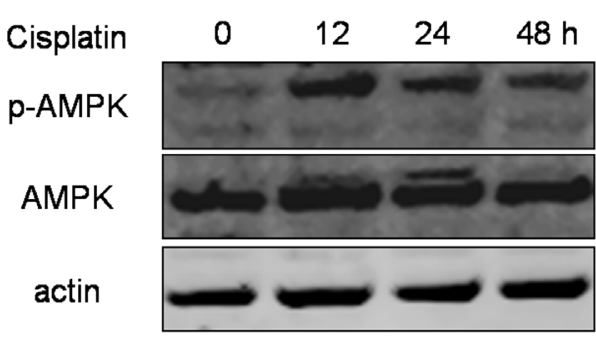

B

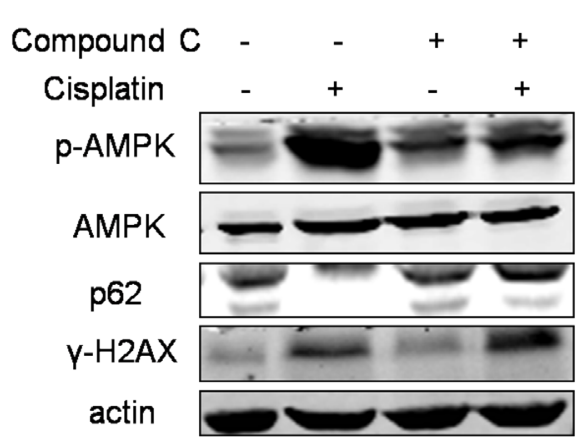

C

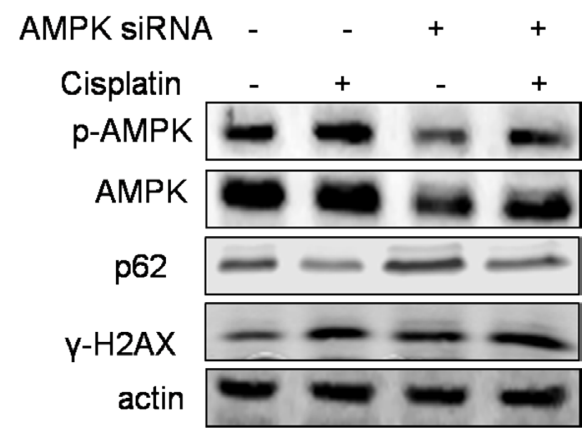

Figure 4. Cisplatin activated AMPK during AKI. A. Primary kidney cells were treated with cisplatin, which was followed by western blot analysis to detect p-AMPK and AMPK. The right panel shows the quantification results for the western blot at various time points. B. Compound $C$ was used to inhibit AMPK in primary kidney cells in the presence or absence of cisplatin. Protein levels of p62, p-AMPK, AMPK, and $\gamma \mathrm{H} 2 \mathrm{AX}$ are shown in the western blot. C. Endogenous AMPK was knocked down by using AMPK SMARTpool siRNA. Protein levels of p-AMPK, AMPK, p62, and $\gamma \mathrm{H} 2 \mathrm{AX}$ were assayed by western blotting. A scrambled siRNA was used as the negative control (-).

\section{DISCUSSION}

In this study, we found that phosphorylation of AMPK regulates autophagy during cisplatininduced AKI (Figure 5). Since p53 can regulate autophagy by inactivating mTOR via AMPK, our results suggest that the p53 pathway may also play a critical role in the pathogenesis of cisplatininduced renal damage (Huber et al., 2012). In line with this view, we found that cisplatin could lead to extensive DNA damage, as evidenced by increasing levels of $\gamma \mathrm{H} 2 \mathrm{AX}$, consistent with the p53-induced DNA damage response (Feng et al., 2005; Vousden and Ryan, 2009). Interestingly, we found that inhibiting autophagy by using chloroquine could not reverse cisplatin-induced renal damage, underlining the critical role of autophagy in AKI. As a physiological response to renal 
stress, autophagy is induced to mitigate the damage caused by chemotherapeutic agents such as cisplatin. Disrupting autophagy in this process, either by using pharmacological inhibitors, such as chloroquine, or by manipulation of the key regulators (e.g., AMPK) in the signaling pathway, can lead to intensified severity during the pathogenesis of renal stress (Figure 5).

\section{In kidney}

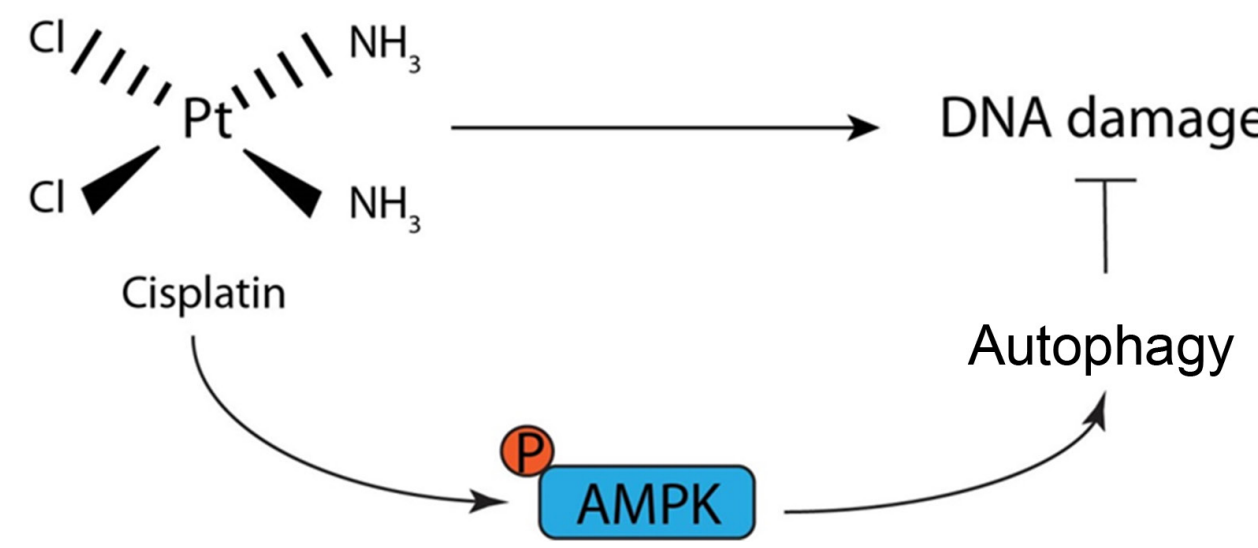

Figure 5. Proposed mechanism for regulation of cisplatin-induced DNA damage during AKI. Current data suggest that the phosphorylation of AMPK regulates autophagy in cisplatin-induced AKI. Cisplatin may lead to extensive DNA damage, as marked by increasing levels of $\gamma \mathrm{H} 2 \mathrm{AX}$. Inhibiting autophagy by treatment with chloroquine worsened the DNA damage caused by cisplatin treatment in the kidney. As a physiological response to renal damage stress, autophagy is induced to protect the kidney from the damage caused by chemotherapeutic agents such as cisplatin. Disrupting autophagy either by using pharmacological inhibitors such as chloroquine or by knockdown of the key regulators (e.g., AMPK) can lead to an intensified severity of AKI.

The role of autophagy in renal stress-related cell death is still debated. Some reports showed that cell death was caused by a high turnover of autophagy during AKI (Suzuki et al., 2008), while other studies suggested that autophagy was induced to protect renal cells from being damaged by chemotherapeutic reagents such as cisplatin (Kaushal et al., 2008). The reason for the discrepancy is not clear, yet we postulate that cell death during cisplatin-induced AKI may be interpreted differently depending on the markers that were adopted in different studies. In our study, we found that increasing levels of $\gamma \mathrm{H} 2 \mathrm{AX}$ were concomitant with decreasing autophagy activity in cisplatin-induced AKI. This result is consistent with the findings of some other reports (Jiang et al., 2010). In line with this view, it is likely that cisplatin induces massive DNA damage, which leads to cell death. As a feedback mechanism, autophagy is induced to compensate for the damage by restoring endangered cellular metabolic levels. As a result, inhibition of autophagy leads to DNA damage in the cell, contributing to the pathogenesis of AKI during cisplatin-induced renal stress. Therefore, autophagy activation may serve as a survival strategy to suppress cisplatin-induced tissue injury.

It is well known that activation of AMPK regulates p53-mediated inactivation of mTOR pathways. Interestingly, AMPK also regulates autophagy activity during cisplatin-induced tissue 
damage. Inhibiting AMPK by using pharmacological inhibitors (compound C) or siRNA led to repressed autophagy in cisplatin-induced AKI, resulting in more DNA damage. Our findings revealed a missing link whereby regulation of autophagy activity directly controls the outcome of cisplatin-induced DNA damage in AKI, suggesting that the p53 pathway also plays a critical role in regulating autophagy during renal pathogenesis.

In future studies, it would be interesting to further dissect the regulatory mechanism of autophagy in stress-induced AKI. Specifically, it will be important to determine whether the regulation of autophagy by AMPK during AKI has pathological relevance to the in vivo system. Generating a genetic knockout model of AMPK in mice may help with evaluating the pathological changes during renal stress. On the other hand, administration of relevant pharmacological inhibitors of AMPK (e.g., compound C) in an in vivo AKI model would also facilitate the demonstration of the regulatory role of AMPK in autophagy. This knowledge, once gained from an appropriately designed genetic model, may greatly increase our understanding of the physiological roles of autophagy in the pathogenesis of renal injuries.

\title{
Conflicts of interest
}

The authors declare no conflict of interest.

\section{ACKNOWLEDGMENTS}

\author{
Research supported by the Science and Technology Department of Hunan Province \\ (Foundation \#2014TT2019).
}

\section{REFERENCES}

Basu A and Krishnamurthy S (2010). Cellular responses to cisplatin-induced DNA damage. J. Nucleic Acids 2010: 201367. Bjorkoy G, Lamark T, Brech A, Outzen H, et al. (2005). p62/SQSTM1 forms protein aggregates degraded by autophagy and has a protective effect on huntingtin-induced cell death. J. Cell Biol. 171: 603-614.

Bolisetty S, Traylor AM, Kim J, Joseph R, et al. (2010). Heme oxygenase-1 inhibits renal tubular macroautophagy in acute kidney injury. J. Am. Soc. Nephrol. 21: 1702-1712.

Feng Z, Zhang H, Levine AJ and Jin S (2005). The coordinate regulation of the p53 and mTOR pathways in cells. Proc. Natl. Acad. Sci. U. S. A. 102: 8204-8209.

Havasi A and Borkan SC (2011). Apoptosis and acute kidney injury. Kidney Int. 80: 29-40.

Huber TB, Edelstein CL, Hartleben B, Inoki K, et al. (2012). Emerging role of autophagy in kidney function, diseases and aging. Autophagy 8: 1009-1031.

Jiang M, Liu K, Luo J and Dong Z (2010). Autophagy is a renoprotective mechanism during in vitro hypoxia and in vivo ischemia-reperfusion injury. Am. J. Pathol. 176: 1181-1192.

Jiang M, Wei Q, Dong G, Komatsu M, et al. (2012). Autophagy in proximal tubules protects against acute kidney injury. Kidney Int. 82: 1271-1283.

Kabeya Y, Mizushima N, Ueno T, Yamamoto A, et al. (2000). LC3, a mammalian homologue of yeast Apg8p, is localized in autophagosome membranes after processing. EMBO J. 19: 5720-5728.

Kaushal GP, Kaushal V, Herzog C and Yang C (2008). Autophagy delays apoptosis in renal tubular epithelial cells in cisplatin cytotoxicity. Autophagy 4: 710-712.

Levine B and Klionsky DJ (2004). Development by self-digestion: molecular mechanisms and biological functions of autophagy. Dev. Cell 6: 463-477.

Miller RP, TadagavadiRK, Ramesh G and Reeves WB (2010). Mechanisms of cisplatin nephrotoxicity. Toxins 2: 2490-2518

Pabla N and Dong Z (2008). Cisplatin nephrotoxicity: mechanisms and renoprotective strategies. Kidney Int. 73: 994-1007.

Pallet N, Bouvier N, Legendre C, Gilleron J, et al. (2008). Autophagy protects renal tubular cells against cyclosporine toxicity. Autophagy 4: 783-791. 
Periyasamy-Thandavan S, Jiang M, Wei Q, Smith R, et al. (2008). Autophagy is cytoprotective during cisplatin injury of renal proximal tubular cells. Kidney Int. 74: 631-640.

Periyasamy-Thandavan S, Jiang M, Schoenlein P and Dong Z (2009). Autophagy: molecular machinery, regulation, and implications for renal pathophysiology. Am. J. Physiol. Renal Physiol. 297: F244-F256.

Price PM, SafirsteinRL and Megyesi J (2009). The cell cycle and acute kidney injury. Kidney Int. 76: 604-613.

Sharfuddin AA and Molitoris BA (2011). Pathophysiology of ischemic acute kidney injury. Nat. Rev. Nephrol. 7: 189-200.

Suzuki C, Isaka Y, Takabatake Y, Tanaka H, et al. (2008). Participation of autophagy in renal ischemia/reperfusion injury. Biochem. Biophys. Res. Commun. 368: 100-106.

Vousden KH and Ryan KM (2009). p53 and metabolism. Nat. Rev. Cancer 9: 691-700.

Yang C, Kaushal V, Shah SV and Kaushal GP (2008). Autophagy is associated with apoptosis in cisplatin injury to renal tubular epithelial cells. Am. J. Physiol. Renal Physiol. 294: F777-F787. 